\title{
SYNTHESIS AND CHARACTERIZATION OF NEW THERMALLY STABLE POLYAMIDES BASED ON 2,5- PYRIDINE DICARBOXYLIC ACID AND AROMATIC DIAMINES
}

\author{
KHALIL FAGHIHI* \\ ${ }^{1}$ Polymer Research Laboratory, Department of Chemistry, Faculty of Science, Islamic Azad University, Branch Arak, Arak, Iran
}

(Received: August 28, 2008 - Accepted: March 19, 2009)

\begin{abstract}
Six new thermally stable polyamides 3a-f were synthesized through the polycondensation reaction of 2,5-pyridine dicarboxylic acid $\mathbf{1}$ with six different derivatives of aromatic diamines 2 a-f in a medium consisting of $\mathrm{N}$-methyl-2-pyrrolidone, triphenyl phosphite, calcium chloride and pyridine. The polycondensation reaction produced a series of novel polyamides containing pyridyl moieties in the main chain in high yield with inherent viscosities between $0.50-0.82 \mathrm{dL} / \mathrm{g}$. The resulting polymers were fully characterized by means of FT-IR spectroscopy, elemental analyses, inherent viscosimetry, and solubility tests. Thermal properties of these polymers were investigated by using thermal gravimetric analysis (TGA) and differantional thermal gravimetry (DTG). All of the polymers were soluble at room temperature in polar solvents such as $\mathrm{N}, \mathrm{N}^{\prime}$-dimethyl acetamide, $\mathrm{N}, \mathrm{N}^{\prime}$-dimethyl formamide, dimethyl sulfoxide and $\mathrm{N}$-methyl-2-pyrrolidone.
\end{abstract}

\section{INTRODUCTION}

Aromatic polyamides have received considerable attention with regard to the production of high performance materials due to their outstanding thermal stability, chemical resistance, electrical and mechanical properties. ${ }^{1-3}$ However their applications are restricted because of their poor solubility in organic solvents and extremely high glass transition temperatures that make them very difficult to be processed by spin coating or thermoforming techniques. ${ }^{4-5}$ Much effort has been made to create structurally modified aromatic polymers having increased solubility and process ability with retention of their high thermal stability. It is known that the solubility of polymers is often increased when flexible bonds such as $\left[-\mathrm{O}-,-\mathrm{SO}_{2}^{-},-\mathrm{CH}_{2}-,-\mathrm{C}\left(\mathrm{CF}_{3}-\right)_{2}\right]$, bulky pendent groups (such as t-butyl, adamantyle and naphtyl), large pendent groups or polar constituents such as heterocyclic segments are incorporated into the polymer backbone due to the altering crystallinity and intermolecular interactions. ${ }^{6-8}$ If the heterocylic moiety is carefully chosen, it is possible to promote solubility without affecting thermal and mechanical properties to any great extent. ${ }^{9-16}$ In our pervious papers we described synthesis of different polyamides and poly(amide-imide)s containing heterocyclic moieties in the main chain such as hydantoin derivatives with improved solubility and thermal properties. ${ }^{17-25}$

In this article, synthesis and characterization of six new polyamides 3a-f containing pyridyl moieties in the main chain was reported from the polycondensation reaction of 2,5-pyridine dicarboxylic acid $\mathbf{1}$ and 1,4phenylene diamine 2a, 1,5-naphthalene diamine 2b, 4,4'-diamino diphenyl ether 2c, 2,6-diamino pyridine 2d, , 4,4'-diamino diphenyl sulfone 2e, 3,3'-diamino diphenyl sulfone $\mathbf{2 f}$ by using $\mathrm{N}$-methyl-2-pyrrolidone (NMP), triphenylphosphite and pyridine as condensing agents. These polymers have a heterocyclic aromatic ring such as pyridyl moiety in the main chain for improving solubility in organic solvents in compared to aromatic polyamides.

\section{EXPERIMENTAL}

Materials

2,5-pyridine dicarboxylic acid 1, 1,4-phenylene diamine 2a, 1,5naphthalene diamine $2 b, 4,4^{\prime}$-diamino diphenylether $2 c, 2,6$-diamino pyridine $2 d, 4,4^{\prime}$-diaminodiphenyl sullfone $2 e, 3,3$ '-diaminodiphenyl sulfone $2 f$, triphenyl phosphate, N-methyl-2-pyrrolidone (NMP), pyridine were purchased from Merck Chemical Company and were used as received. Commercially available calcium chloride $\left(\mathrm{CaCl}_{2}\right.$; from Merck) was dried under vacuum at $150^{\circ} \mathrm{C}$ for $6 \mathrm{~h}$

Techniques

Fourier transform infrared (FTIR) spectra were recorded on Galaxy series FTIR 5000 spectrophotometer (England). Spectra of solids were performed by using $\mathrm{KBr}$ pellets. Vibrational transition frequencies are reported in wave number $\left(\mathrm{cm}^{-1}\right)$. Band intensities are assigned as weak (w), medium (m), shoulder (sh), strong (s) and broad (br). Inherent viscosities were measured by a standard procedure by using a Technico Regd Trad Merk Viscometer. Thermal Gravimetric Analysis (TGA and DTG) data for polymers were taken on a Mettler TA4000 System under $\mathrm{N}_{2}$ atmosphere at a rate of $10^{\circ} \mathrm{C} / \mathrm{min}$. Elemental analyses were performed by the Arak Petrochemical Company, Arak, Iran.

Polymer synthesis

The PAs 3a-f were prepared by the following general procedure (using polymer 3a as an example). Into a $25 \mathrm{~mL}$ round-bottomed flask which was fitted with a stirring bar were placed 1,4-phenylene diamine $\mathbf{2 a}(0.069 \mathrm{~g}, 0.64$ $\mathrm{mmol})$, diacid 1 (0.106 g, $0.64 \mathrm{mmol})$, calcium chloride $(0.20 \mathrm{~g}, 1.80 \mathrm{mmol})$, triphenyl phosphite $(1.68 \mathrm{~mL}, 6.00 \mathrm{mmol})$, pyridine $(0.36 \mathrm{~mL})$ and $\mathrm{N}$-methyl2-pyrolidone $(1.6 \mathrm{~mL})$. The reaction mixture was heated under reflux on an oil bath at $60^{\circ} \mathrm{C}$ for $1 \mathrm{~h}$, then at $90^{\circ} \mathrm{C}$ for $2 \mathrm{~h}$, and at $130^{\circ} \mathrm{C}$ for $8 \mathrm{~h}$. Then the reaction mixture was poured into $25 \mathrm{~mL}$ of methanol and the precipitated polymer was collected by filtration and washed thoroughly with methanol and dried at $60^{\circ} \mathrm{C}$ for $12 \mathrm{~h}$ under vacuum to leave $0.14 \mathrm{~g}(91.5 \%)$ of white solid polymer $3 \mathrm{a}$. The PAs 3a-f were analyzed by using FT-IR spectroscopy.

\section{RESULTS AND DISCUSSION}

\section{Polymer synthesis}

PAs 3a-f were synthesized by the direct polycondensation reaction of an equimolar mixture of diacid $\mathbf{1}$ with six aromatic diamines 2a-f in a medium consisting of N-methyl-2-pyrrolidone, triphenyl phosphite, calcium chloride and pyridine (Scheme 1).

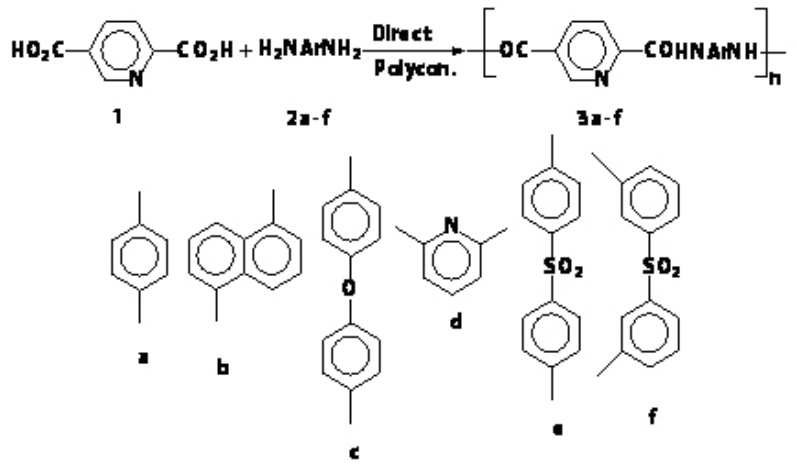

Scheme 1 Synthesis of polyamides 3a-f

The syntheses and some physical properties of these new PAs 3a-f are given in Table 1 . The entire polycondensation reaction readily proceeded in a homogeneous solution while tough and stringy precipitates formed as the viscous PAs solution was obtained in good yields.

Table 1.- Some physical properties of PAs 3a-f

\begin{tabular}{|c|c|c|c|c|}
\hline Diamines & Polymer & Yield (\%) & $\eta_{\text {inh }}(\mathrm{dL} / \mathrm{g})^{\text {a }}$ & Colors \\
\hline 2a & 3a & 91 & 0.70 & White \\
\hline 2b & 3b & 92 & 0.65 & Off-white \\
\hline 2c & 3c & 89 & 0.50 & White \\
\hline 2d & 3d & 85 & 0.70 & Off-White \\
\hline 2e & 3e & 90 & 0.82 & Yellow \\
\hline 2f & 3f & 87 & 0.72 & Yellow \\
\hline
\end{tabular}

${ }^{a}$ Measured at a concentration of $0.5 \mathrm{~g} / \mathrm{dL}$ in DMF at $25^{\circ} \mathrm{C}$. Measured at Concentration of $0.5 \mathrm{~g} / \mathrm{dL}$ in $\mathrm{DMF}$ at $25^{\circ} \mathrm{C}$. 
Polymer characterization

The syntheses and some physical properties of PAs 3a-f are summarized in Table 1. These polymers had inherent viscosities around $0.50-0.82 \mathrm{dL} / \mathrm{g}$ and showed white to yellow crystals. These polymers were confirmed to be PAs with FT-IR spectroscopy and elemental analyses (Table 2). A representative FT-IR spectrum of polymer 3a shows that the carbonyl peak of polymer shift to lower frequency in comparison with diacid 1 and $\mathrm{OH}$ peak at $2500-3100 \mathrm{~cm}^{-1}$ of diacid disappeared. The polymer exhibited characteristic absorption bands at $1709 \mathrm{~cm}^{-1}$ for the amide carbonyl group and absorption bands of amide groups appeared at $3308 \mathrm{~cm}^{-1}$ (N-H stretching). Also spectral data of resulting PA 3a-f were showed in Table 2. All of the polymers show similar FT-IR spectrum.

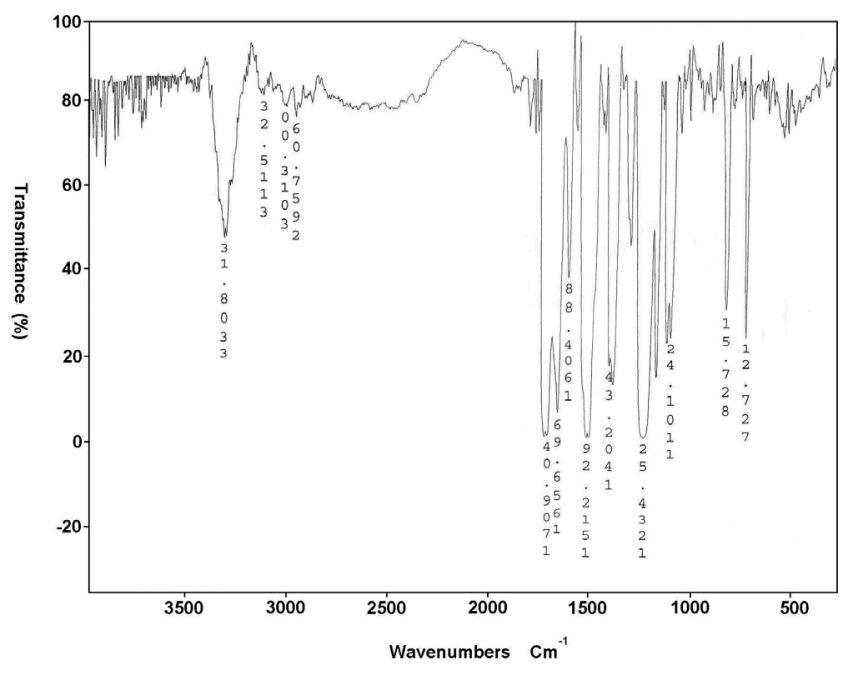

Fig. 1 FTIR Spectrum of PA 3a

Table 2.- FTIR Characterization of PAIs 3a-f

\begin{tabular}{|c|c|}
\hline Polymer & Spectral data \\
\hline $3 a$ & $\begin{array}{l}\text { FT-IR (KBr): } 3308(\mathrm{~m}), 3115(\mathrm{w}), 3013(\mathrm{w}), 2957(\mathrm{w}), 1709 \\
(\mathrm{~s}), 1656(\mathrm{~s}), 1604(\mathrm{~m}), 1512(\mathrm{~s}), 1402(\mathrm{~s}), 1234(\mathrm{~s}), 1101(\mathrm{~m}), \\
827(\mathrm{~m}), 727(\mathrm{~m}) \mathrm{Cm}^{-1} .\end{array}$ \\
\hline $3 b$ & $\begin{array}{l}\text { FT-IR (KBr): } 3325(\mathrm{~m}), 1698(\mathrm{~m}), 1589(\mathrm{~s}), 1512(\mathrm{~s}), 1400 \\
(\mathrm{~m}), 1317(\mathrm{~s}), 1242(\mathrm{~m}), 1182(\mathrm{w}), 1147 \text { (m), } 1107(\mathrm{~m}), 1049 \\
(\mathrm{w}), 727(\mathrm{w}) \mathrm{Cm}^{-1} .\end{array}$ \\
\hline $3 c$ & $\begin{array}{l}\text { FT-IR (KBr): } 3308(\mathrm{~m}), 3055(\mathrm{w}), 1651(\mathrm{~m}), 1604(\mathrm{~m}), \\
1500(\mathrm{~s}), 1408(\mathrm{~m}), 1319(\mathrm{~m}), 1257(\mathrm{~m}), 1221(\mathrm{~m}), 1103(\mathrm{w}), \\
1022(\mathrm{w}), 846(\mathrm{w}), 761(\mathrm{w}) \mathrm{Cm}^{-1}\end{array}$ \\
\hline 3d & $\begin{array}{l}\text { FT-IR (KBr): } 3335(\mathrm{~m}), 3055(\mathrm{w}), 1705(\mathrm{~m}), 1606(\mathrm{~m}), 1516 \\
(\mathrm{~s}), 1408(\mathrm{~m}), 1319(\mathrm{~m}), 1265(\mathrm{~m}), 1184(\mathrm{w}), 1116(\mathrm{w}), 993 \\
(\mathrm{w}), 895(\mathrm{w}), 844(\mathrm{w}), 792(\mathrm{w}), 761(\mathrm{w}), 688(\mathrm{w}) \mathrm{Cm}^{-1} .\end{array}$ \\
\hline $3 \mathbf{e}$ & $\begin{array}{l}\text { FT-IR (KBr): } 3310(\mathrm{~m}), 1700(\mathrm{~s}), 1601(\mathrm{~m}), 1521(\mathrm{~s}), 1489 \\
(\mathrm{~s}), 1408(\mathrm{~m}), 1325(\mathrm{~m}), 1269(\mathrm{~m}), 1184(\mathrm{w}), 1072(\mathrm{w}), 966 \\
(\mathrm{w}), 848(\mathrm{w}), 783(\mathrm{w}), 688(\mathrm{w}) \mathrm{Cm}^{-1} .\end{array}$ \\
\hline $3 f$ & $\begin{array}{l}\text { FT-IR (KBr): } 3331(\mathrm{~m}), 3076(\mathrm{w}), 1699(\mathrm{~s}), 1606(\mathrm{~s}), 1523 \\
(\mathrm{~s}), 1411(\mathrm{~s}), 1321(\mathrm{~s}), 1267(\mathrm{~s}), 1178(\mathrm{~s}), 1076(\mathrm{w}), 1024(\mathrm{w}), \\
947(\mathrm{w}), 854(\mathrm{~m}), 769(\mathrm{~m}), 688(\mathrm{~m}) \mathrm{Cm}^{-1} .\end{array}$ \\
\hline
\end{tabular}

The elemental analysis of the resulting polymers is in good agreement with the calculated values for the proposed structures (Table 3).
Table 3.- Elemental Analysis of PAs 3a-f.

\begin{tabular}{|c|c|c|c|c|c|}
\hline Polymer & Formula & & $\mathrm{C} \%$ & $\mathrm{H} \%$ & N\% \\
\hline $3 \mathbf{a}$ & $\begin{array}{c}\mathrm{C}_{13} \mathrm{H}_{9} \mathrm{~N}_{3} \mathrm{O}_{2} \\
(239.13)_{\mathrm{n}}\end{array}$ & $\begin{array}{l}\text { Calcd } \\
\text { Found }\end{array}$ & $\begin{array}{l}65.29 \\
64.90\end{array}$ & $\begin{array}{l}3.76 \\
3.10\end{array}$ & $\begin{array}{l}17.56 \\
17.00\end{array}$ \\
\hline $3 b$ & $\begin{array}{c}\mathrm{C}_{17} \mathrm{H}_{11} \mathrm{~N}_{3} \mathrm{O}_{2} \\
(289.17)_{\mathrm{n}}\end{array}$ & $\begin{array}{l}\text { Calcd } \\
\text { found }\end{array}$ & $\begin{array}{l}70.60 \\
70.00\end{array}$ & $\begin{array}{l}3.80 \\
3.40\end{array}$ & $\begin{array}{l}14.52 \\
13.80\end{array}$ \\
\hline $3 c$ & $\begin{array}{c}\mathrm{C}_{17} \mathrm{H}_{13} \mathrm{~N}_{3} \mathrm{O}_{3} \\
(307.17)_{\mathrm{n}}\end{array}$ & $\begin{array}{l}\text { Calcd } \\
\text { found }\end{array}$ & $\begin{array}{l}66.24 \\
65.90\end{array}$ & $\begin{array}{l}4.23 \\
4.00\end{array}$ & $\begin{array}{l}13.67 \\
13.10\end{array}$ \\
\hline 3d & $\begin{array}{c}\mathrm{C}_{12} \mathrm{H}_{8} \mathrm{~N}_{4} \mathrm{O}_{2} \\
(240.12)_{\mathrm{n}}\end{array}$ & $\begin{array}{l}\text { Calcd } \\
\text { found }\end{array}$ & $\begin{array}{l}60.01 \\
59.20\end{array}$ & $\begin{array}{l}3.33 \\
2.90\end{array}$ & $\begin{array}{l}23.32 \\
22.80\end{array}$ \\
\hline $3 e$ & $\begin{array}{c}\mathrm{C}_{19} \mathrm{H}_{13} \mathrm{~N}_{3} \mathrm{O}_{4} \mathrm{~S} \\
(379.19)_{\mathrm{n}}\end{array}$ & $\begin{array}{l}\text { Calcd } \\
\text { found }\end{array}$ & $\begin{array}{l}60.17 \\
59.80 \\
\end{array}$ & $\begin{array}{l}3.42 \\
3.00 \\
\end{array}$ & $\begin{array}{l}11.07 \\
10.60\end{array}$ \\
\hline $3 f$ & $\begin{array}{c}\mathrm{C}_{19} \mathrm{H}_{13} \mathrm{~N}_{3} \mathrm{O}_{4} \mathrm{~S} \\
(379.19)_{\mathrm{n}}\end{array}$ & $\begin{array}{l}\text { Calcd } \\
\text { found }\end{array}$ & $\begin{array}{l}60.17 \\
59.60\end{array}$ & $\begin{array}{l}3.42 \\
2.90\end{array}$ & $\begin{array}{l}11.07 \\
10.70\end{array}$ \\
\hline
\end{tabular}

The solubility of PAs 3a-f was investigated with $0.01 \mathrm{~g}$ polymeric samples in $2 \mathrm{~mL}$ of solvent. All of the polymers are dissolved in organic solvents such as DMF, DMAC, DMSO and NMP at room temperature and are insoluble in solvents such as chloroform, methylene chloride, methanol, ethanol and water (Table 4).

Table 4.- Solubility behavior PAs 3a-f

\begin{tabular}{|c|c|c|c|c|c|}
\hline Solvents & $\mathbf{3 a}$ & $\mathbf{3 b}$ & $\mathbf{3 c}$ & $\mathbf{3 d}$ & $\mathbf{3 e}$ \\
\hline $\mathrm{DMAc}$ & + & + & + & + & + \\
\hline $\mathrm{DMSO}$ & + & + & + & + & + \\
\hline $\mathrm{DMF}$ & + & + & + & + & + \\
\hline $\mathrm{NMP}$ & + & + & + & + & + \\
\hline $\mathrm{THF}$ & + & + & + & + & + \\
\hline $\mathrm{CHCl}$ & - & - & - & - & - \\
\hline $\mathrm{Acetone}$ & - & - & - & - & - \\
\hline $\mathrm{EtOH}_{2}$ & - & - & - & - & - \\
\hline $\mathrm{MeOH}_{2} \mathrm{CH}$ & - & - & - & - & - \\
\hline $\mathrm{CH}_{2} \mathrm{Cl}$ & - & - & - & - & - \\
\hline $\mathrm{H}_{2} \mathrm{O}$ & - & - & - & - & - \\
\hline
\end{tabular}

+: Soluble at room temperature, -: Insoluble at room temperature.

Thermal properties

The thermal properties of PAs 3a-f were investigated with TGA and DTG in a nitrogen atmosphere at a heating rate of $10^{\circ} \mathrm{C} / \mathrm{min}^{1}$ and the thermal data 
are summarized in Table 5. The initial decomposition temperatures of 5 and $10 \%$ weight losses $\left(\mathrm{T}_{5}\right.$ and $\left.\mathrm{T}_{10}\right)$ and the char yield at $600^{\circ} \mathrm{C}$ for these polymers are summarized in Table 5 . These polymers exhibited good resistance to thermal decomposition, up to $295-315^{\circ} \mathrm{C}$ in nitrogen, and began to decompose gradually above that temperature. $\mathrm{T}_{5}$ for these polymers ranged from 295 to 315 ${ }^{\circ} \mathrm{C}$ and $\mathrm{T}_{10}$ for all polymers ranged from 325 to $365^{\circ} \mathrm{C}$, and the residual weight for these polymers at $600{ }^{\circ} \mathrm{C}$ ranged from 10 to $58 \%$ in nitrogen. Results show that two PAs $\mathbf{3 e}$ and $\mathbf{3} \mathbf{f}$ with sulfone moiety in the main chain have higher thermal stability in comparing to other PAs 3a-d because they have a rigid structure such as sulfone moiety in the main chain. These results show PAs 3a-f have thermal resistance and they can be used as engineering plastic in many applications.

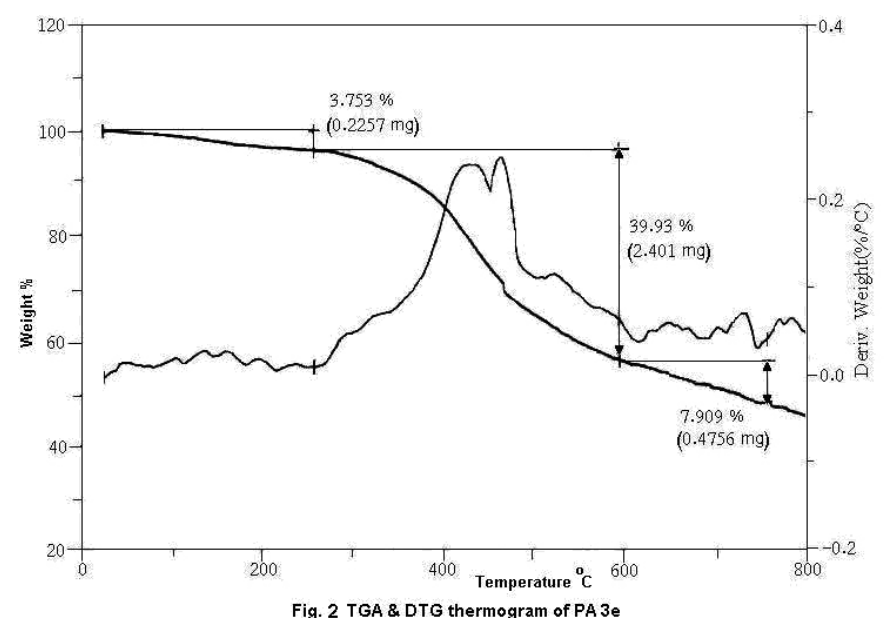

Table 5.- Thermal behavior of PAs $\mathbf{3 a - f}$

\begin{tabular}{|c|c|c|c|}
\hline Polymer & $\mathrm{T}_{5}\left({ }^{\circ} \mathrm{C}\right)^{\mathrm{a}}$ & $\mathrm{T}_{10}\left({ }^{\circ} \mathrm{C}\right)^{\mathrm{b}}$ & $\begin{array}{c}\text { Char Yield } \\
\mathrm{c}\end{array}$ \\
\hline 3a & $305-310$ & $325-330$ & $10 \%$ \\
\hline 3b & $295-300$ & $350-355$ & $33 \%$ \\
\hline 3c & $305-310$ & $330-335$ & $17 \%$ \\
\hline 3d & $305-310$ & $360-365$ & $55 \%$ \\
\hline 3e & $315-320$ & $365-370$ & $58 \%$ \\
\hline 3f & $300-305$ & $345-350$ & $48 \%$ \\
\hline
\end{tabular}

a,b Temperature at which $5 \%$ and $10 \%$ weight loss was recorded by TGA at heating rate of $10^{\circ} \mathrm{C} / \mathrm{min}$ in $\mathrm{N}_{2}$ respectively ${ }^{\circ}$ Percentage weight of material left undecomposed after TGA analysis $600^{\circ} \mathrm{C}$.

\section{CONCLUSION}

This work involved the syntheses of several new PAs 3a-f through the direct polycondensation reaction of diacid $\mathbf{1}$ with six aromatic diamines $\mathbf{2 a - f}$ by using triphenyl phosphite, NMP, calcium chloride and pyridine as condensing agents. These new PAs were soluble in various organic solvents and had good thermal stability. The presence of pridyl segments as a hetrocyclic ring into the backbone increased the solubility of these polymers. These properties could make these PAs attractive for practical applications, such as processable highperformance engineering plastics.

\section{REFERENCES}

1. Cassidy, P. E. Thermally Stable Polymers, Synthesis and Properties; Dekker: New York (1980).

2. Park, K. P.; Kakimoto, M. A.; Imai, Y. J. Polym. Sci. Part A: Polym. Chem., 33, 1031, (1995).

3. Yang, C. P.; Hsiao, S. H.; Yang, C. C. J. Polym. Sci. Part A: Polym. Chem., 35, 2147, (1997).

4. Hendrick, J. L.; Twieg, R. Macromolecules, 25, 2021, (1992).

5. Choi, K. H.; Jung, J. C. Macromol. Mater. Eng., 289, 737, (2004).

6. Yang, C. P.; Hsiao, S. H.; Chung, C. L. Polym. Int., 54, 716, (2005).

7. Hsiao, S. H.; Yang, C. P.; Huang, S. C. J. Polym. Sci. Part A: Polym. Chem., 42, 2377, (2004).

8. Wang, X. L.; Li, Y. F.; Gong, C. L.; Ma, T.; Yang, F. C. J. of Fluorine Chem., 129, 56, (2008).

9. Li, N.; Cui, Z.; Zhang, S.; Xing, W. Polym. 48, 7255, (2007).

10. Hamciuc, E.; Hamciuc, C.; Cazacu, M. Eur. Polym. J., 43, 4739, (2007).

11. Zhang, Q.; Li, S.; Li, W.; Zhang, S. Polym., 48, 624, (2007).

12. Zhao, X.; Li, Y. F.; Zhang, S. J.; Shao, Y.; Wang, X. L. Polym., 48, 5241, (2007).

13. Zhu, Y.; Zhao, P.; Cai, X.; Meng, W. D.; Qing, F. L. Polym., 48, 3116, (2007).

14. Mehdipour Ataei, S.; Bahri Laleh, N.; Amirshaghaghi, A. Polym. Degrad. \& Stability, 91, 2622, (2006)

15. Lee, S. B.; Shin, G. J.; Chi, J. H.; Zin, W. C.; Jung, J. C.; Hahm, S. G.; Ree, M.; Chang, T. Polym., 47, 6606, (2006).

16. Mehdipour Ataei, S.; Arabi, H.; Bahri Laleh, N. Eur. Polym. J., 42, 2343, (2006).

17. Faghihi, Kh.; Hajibeygi, M. Eur. Polym. J., 39, 2307, (2003).

18. Faghihi, Kh.; Zamani, Kh.; Mirsamie, A.; Mallakpour, S. E. J. Appl. Polym. Sci., 91, 516, (2004).

19. Faghihi, Kh.; Zamani, Kh.; Mirsamie, A.; Mallakpour, S. E. Polym. Int., 53, 126, (2004)

20. Faghihi, Kh.; Hajibeygi, M. Macromol. Res., 13, 14, (2005).

21. Faghihi, Kh.; Naghavi, H. J. Appl. Polym. Sci., 96, 1776, (2005).

22. Faghihi, Kh. Polym. J., 37, 449, (2005).

23. Faghihi, Kh. J. Appl. Polym. Sci., 102, 5062, (2006).

24. Faghihi, Kh.; Mozaffari, Z. J. Appl. Polym. Sci., 108, 1152, (2008).

25. Faghihi, Kh.; Naghavi, H. J. Appl. Polym. Sci., 108, 1136, (2008). 